\section{Extraction of Seeds of Hot Peppers Using a Modified Meat Mincer}

\author{
D.C.S. Tay' \\ Genetic Resources and Seed Unit, Asian Vegetable Research and \\ Development Center, P. O. Box 42, Shanhua, Tainan, Taiwan, ROC
}

Seeds of hot peppers may be extracted manually by cutting and splitting the fruit longitudinally and scratching out the seeds with a small blade or similar tool. This method is tedious and causes irritations to the workers, especially from the small and pungent types. Alternatively, fruits may be dried artificially either in bulk or still attached to the plants, with seeds being separated later by threshing. However, seed cleaning is difficult and causes irritations because of the fine pungent dust resulting from threshing.

A wet seed extraction method, described below, using a modified household meat mincer was adopted at the Asian Vegetable Research and Development Center (AVRDC). This method is very efficient as compared to manual extraction, and it also alleviates the irritation problems caused by both the wet and dry extraction methods.

A mincer (Fig. 1), with a cutting area of $6.5 \mathrm{~cm}$ in diameter, can grind up to $100 \mathrm{~kg}$ of pepper fruits (average size of $2.0 \mathrm{~cm}$ wide $\times 15.0 \mathrm{~cm}$ long) per hour at AVRDC. The manual method would require $\approx 360 \mathrm{~h}$ to extract the same amount of pepper fruits.

Blunting, by filing down the sharp rotating cutting blades, is the only modification (Fig. 2). It is important to blunt all the sharp edges of the blades because even a few remaining sharp edges will cut or damage the seeds during grinding. The stationary cutting plate with large perforations (hole diameter $=13.5 \mathrm{~mm}$ ) and the plate with small ones (hole diameter $=9.5 \mathrm{~mm}$ ) are recommended for grinding large and small fruits, respectively, to minimize seed damage (Fig. 2).

Grinding is done without adding water and without removing the fruit peduncles. The ground fruits are collected in a plastic bucket for washing. After each lot is ground, the mincer, including the cutting components, has to be washed free of any remaining seed and fruit pulp to prevent contamination of the next lot.

As the ground pepper fruit with seeds is pungent, workers should wear rubber kitchen gloves during washing. No fermentation is required. The ground pepper pulp is poured

Received for publication 7 Mar. 1990. The cost of publishing this paper was defrayed in part by the payment of page charges. Under postal regulations, this paper therefore must be hereby marked advertisement solely to indicate this fact. 'Associate Seed Technologist and Head. (right) Machine is ready for grinding. be decanted using ordinary household 15liter plastic buckets. Avoid using white or straw-colored buckets so that any seed inadvertently left in the bucket can be seen and washed off. The clean seeds may be treated against seedborne viruses with a $10 \%$ trisodium phosphate solution for $2 \mathrm{~h}$ (Rast and Stijger, 1987)., The seeds are then put into nylon-net bags, placed in an ordinary laundry spin-dryer to remove free water and then spread out for drying in the sun or in an oven at a temperature not exceeding 40C. The cleaned seed may be rinsed in the pepper juice before spin-drying to improve the seed color after drying. Two mincers may be used in alternation to prevent overheating of the mincer's motor.

\section{Literature Cited}

fruit pulp are decanted until only good clean seeds remain. For a larger volume, washing with a flotation sluice to remove the lighter debris may be used. Otherwise, the lot may have to be washed in small batches to facilitate recantation. Small batches easily can
Rast, A.T.B. and C.M. Stijger. 1987. Disinfection of pepper seed infected with different strains of Capsicum mosaic viruses by trisodium phosphate and dry heat treatment. Plant Pathol. 36:583-588.

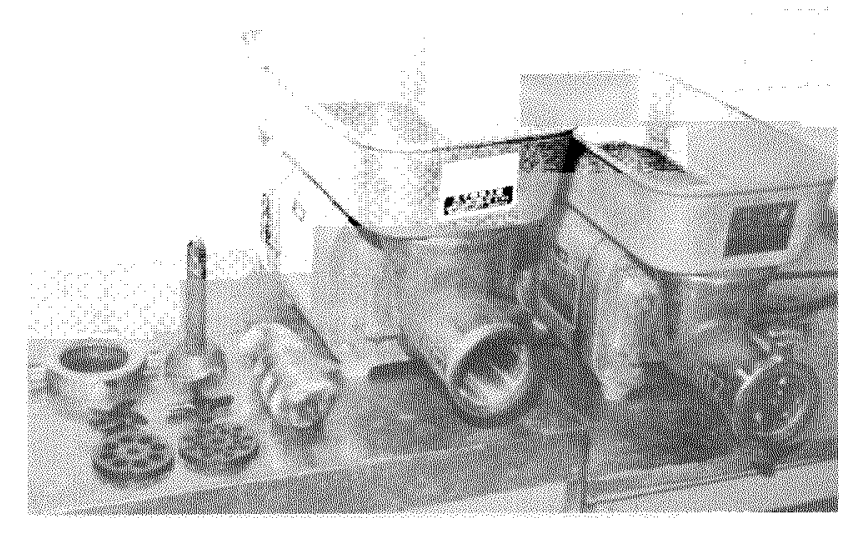

Fig. 1. Meat mincers used at AVRDC. (left) Machine is dismantled to show the grinding components.

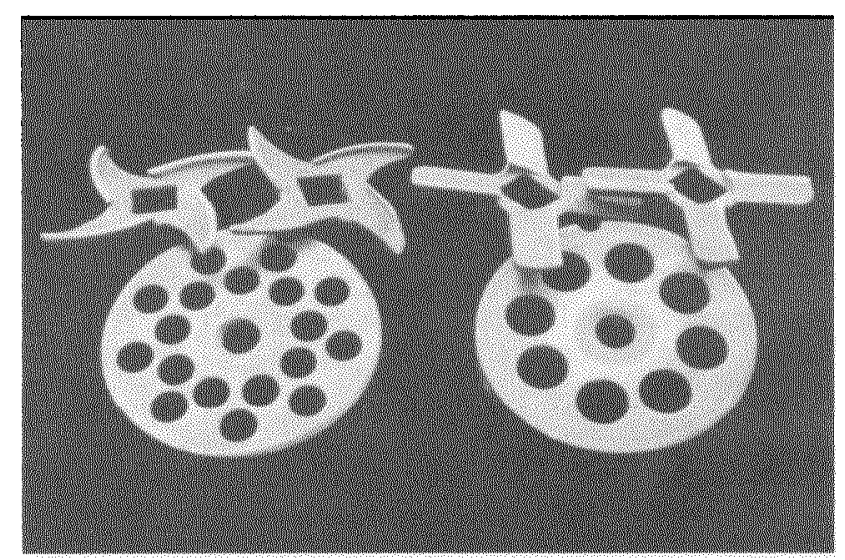

Fig. 2. Cutting components of the mincers showing two types of rotating cutting blades (top) and two types of stationary perforated cutting plates (below). The rotating cutting blade on the left of each type is the original blade; the one on the right is the modified filed-down blade. The stationary perforated cutting plate with the 9.5 -mm-diameter perforations (left) is used for grinding small fruits. The plate with the 13.5 -mm-diameter perforations (right) is used for grinding large fruits. 